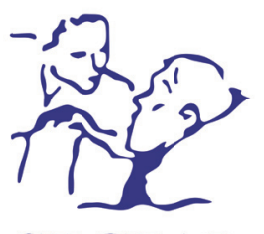

Medicina Paliativa

www.medicinapaliativa.es

ORIGINAL

\title{
Malestar emocional y estrategias de afrontamiento en pacientes avanzados ante la situación de final de vida
}

\author{
Eva Rodríguez Bruzos*, Clara Fraguell Hernando, Mercè Navarro Rodríguez, \\ Anna Escolà Fustero, Helena Villar Abelló, Sílvia de Quadras Roca, Marta Argilés Huguet \\ y Meritxell Naudeillo Cosp
}

Equipos de Atención Psicosocial Mutuam Barcelona

Recibido el 18 de febrero de 2018

Aceptado el 28 de julio de 2018

\author{
PALABRAS CLAVE \\ Malestar emocional, \\ afrontamiento, \\ adaptación, final de \\ vida, paciente \\ avanzado, cuidados \\ paliativos.
}

\begin{abstract}
Resumen
Introducción: El final de vida constituye una situación de gran impacto emocional para pacientes y familiares. En estas circunstancias los pacientes ponen en marcha diferentes estrategias de afrontamiento para poder hacer frente a esta difícil y compleja situación.

Objetivos: Conocer el grado de malestar emocional de los pacientes en situación de final de vida, explorar las estrategias de afrontamiento que utilizan y comparar el malestar emocional y las estrategias que emplean los pacientes atendidos en un centro hospitalario con las de los pacientes atendidos en su domicilio.

Método: Estudio transversal correlacional. La muestra está constituida por 75 pacientes en situación de final de vida, el 53,3 \% fueron tratados en el domicilio y el 46,7 \% en un centro hospitalario. Se realizó una entrevista individual con cada paciente donde se recogían sus datos sociodemográficos y clínicos y se administraban los cuestionarios de malestar emocional (DME) y de estrategias de afrontamiento (MINI-MAC y BRCS).

Resultados: El 73,3\% de los pacientes presentan malestar emocional y el 81,3\% expresan preocupaciones relacionadas principalmente con la familia (47,5\%). Las estrategias de afrontamiento más utilizadas son el espíritu de lucha $(45,9 \%$ y la resiliencia (44\%). Cuando se comparan ambos grupos, los pacientes atendidos en el domicilio presentan puntuaciones significativamente más elevadas en las estrategias de preocupación ansiosa $(p=0,016)$ y desesperanza $(p=0,005)$, respecto a los pacientes ingresados en un centro hospitalario.
\end{abstract}

\footnotetext{
*Autor para correspondencia:

Eva Rodríguez Bruzos

Equipos de Atención Psicosocial Mutuam Barcelona

C/ Mare de Déu de la Salut, 49. 08024 Barcelona

Correo electrónico: evarb71@hotmail.com
}

DOI: 10.20986/medpal.2019.1062/2019

1134-248X/ ( 2019 Sociedad Española de Cuidados Paliativos. Publicado por Inspira Network. Todos los derechos reservados. 
Conclusiones: A pesar de que son pacientes que presentan malestar emocional, una importante proporción de ellos utiliza estrategias de afrontamiento adaptativas. No obstante, la elevada proporción de malestar entre los pacientes del estudio nos sugiere la importancia de desarrollar intervenciones psicológicas preventivas para disminuir este malestar y proporcionar estrategias adaptativas que mejoren la calidad de vida del paciente al final de la vida.

\section{KEYWORDS}

Emotional distress, coping, adjustment, end of life, advanced patient, palliative care.

\begin{abstract}
Introduction: The end of life represents a situation with a high emotional impact on patients and family members. Under these circumstances, patients set in motion a number of coping strategies to deal with this complex, difficult situation.

Objectives: To assess emotional distress levels in patients at the end of life, to explore the coping strategies they use, and to compare emotional distress and coping strategies between home-care and hospitalized subjects.

Method: This was a correlational, cross-sectional study. The sample included 75 patients at the end of life. Of these, $53.3 \%$ were being cared for in their homes, and $46.7 \%$ in health facilities. A one-on-one interview was carried out with each patient to collect sociodemographic and clinical data, and to administer emotional distress (DME) and coping strategies (MINI-MAC and BRCS) questionnaires.

Results: A total of $73.7 \%$ of patients have emotional distress, and $81.3 \%$ report concerns, primarily about their families (47.5\%). The most commonly used coping strategies include a fighting spirit $(45.5 \%)$ and resilience $(44 \%)$. Patients cared for at home show significantly higher anxious worrying $(p=0.016)$ and hopelessness $(p=0.005)$ scores when compared to hospitalized individuals.

Conclusions: Despite their having emotional distress, a significant proportion of these patients develop adaptive coping strategies. However, the high rate of distress found among study patients suggests the importance of developing preventive psychological interventions to reduce such distress and provide adaptive strategies to improve quality of life at the end of life.
\end{abstract}

\section{Introducción}

La fase de final de vida es una de las experiencias vitales más difíciles, emocionalmente impactante y de importantes necesidades que tienen que afrontar las personas. En esta situación de gran intensidad emocional pueden aparecer tristeza, depresión, ansiedad, desesperanza, miedos, preocupaciones, aislamiento y agotamiento emocional, entre otra sintomatología psicológica, que pueden disminuir de forma importante la calidad de vida del paciente ${ }^{1,2}$.

Algunos datos indican que el 58,3\% de los pacientes en fase de final de vida presentan malestar emocional moderado $^{3}$. Según un estudio europeo, la prevalencia de malestar emocional en pacientes en la fase de final de vida es del $60 \% 4$. Esta situación puede generar un fuerte impacto emocional no únicamente en el paciente, sino también en su entorno familiar y social y en el equipo asistencial ${ }^{5-7}$.

En esta fase de la enfermedad, tanto el paciente como sus familiares pasan por momentos muy complicados y de gran sufrimiento emocional, lo que requiere un importante esfuerzo de adaptación.
Las reacciones de los pacientes pueden ser muy variadas ante los mismos acontecimientos y no todos los pacientes se adaptan a esta situación, lo cual facilita la aparición o empeoramiento de los problemas emocionales. Por lo tanto, las dificultades de adaptación son muy frecuentes en estos pacientes $^{8}$.

Existen diversas variables sociodemográficas, clínicas y emocionales que pueden modular la adaptación a la experiencia de final de vida: la presencia de síntomas, su intensidad y control, la información que tiene el paciente, la edad, las variables de personalidad, las creencias, la disponibilidad de apoyo familiar y social, las estrategias de afrontamiento, el locus de control, las expectativas, etc. ${ }^{9,10}$.

De estas variables, el trabajo se centrará en el estudio de las estrategias de afrontamiento, entendiendo estas, según el modelo de Lazarus y Folkman ${ }^{11}$, como la cantidad de medios de que dispone el paciente para hacer frente al estrés que produce la situación de final de vida, de la intensidad y habilidad con los que utiliza estos medios y de la eficacia intrínseca de los mismos. 
Las estrategias de afrontamiento pueden ser estrategias adaptadas a la situación y estrategias no adaptadas a la situación de enfermedad. Entre las primeras se incluye: la búsqueda de apoyo emocional y social, la actitud de espíritu de lucha y la aceptación, combinadas con los esfuerzos por mantener el autocontrol sobre la enfermedad. Las estrategias menos adaptativas incluirían una actitud de desesperanza y resignación, combinada con una actitud pasiva y de evitación ${ }^{12-14}$.

Existen pocos estudios en nuestro entorno que valoren el estado emocional y las estrategias de afrontamiento de los pacientes en situación de final de vida, y no se han hallado estudios realizados en población española que valoren las estrategias de afrontamiento en función de la ubicación donde son atendidos.

Por estos motivos, en el presente trabajo se plantean los siguientes objetivos: 1) explorar el malestar emocional de los pacientes en situación de final de vida que recibieron atención por el equipo de psicólogas del Equipo de Atención Psicosocial (EAPS) Mutuam de Barcelona en los diferentes recursos; 2) conocer sus estrategias de afrontamiento y analizar si existen diferencias, teniendo en cuenta estas variables, entre los pacientes tratados en su domicilio con un servicio de atención domiciliaria (PADES) y los pacientes atendidos en un entorno hospitalario (hospital, centro sociosanitario), y 3) conocer si las variables psicológicas de malestar emocional y estrategias de afrontamiento se correlacionan entre sí.

\section{Material y método}

\section{Diseño}

Estudio transversal correlacional. Los datos se recogieron entre enero y octubre de 2016, a través de la entrevista clínica y la administración de cuestionarios para evaluar el malestar emocional y las estrategias de afrontamiento de los pacientes participantes en el estudio.

\section{Participantes}

Pacientes en condición clínica avanzada, en situación de final de vida, con un tiempo pronóstico de vida inferior a 6 meses, atendidos por el equipo de psicólogas del EAPS Mutuam Barcelona en los diferentes recursos de atención: domicilio, hospital y centro sociosanitario.

Los criterios de inclusión utilizados en el presente estudio fueron los siguientes: pacientes adultos mayores de 18 años, con un pronóstico de vida inferior a 6 meses y pacientes con conciencia pronóstica.

Y como criterios de exclusión: pacientes afectados con patologías mentales graves; pacientes con deterioro cognitivo moderado/grave; pacientes en agonía y/o situación de últimos días, y pacientes extranjeros con dificultades para la comprensión del idioma castellano.

Ciento cinco pacientes en situación de final de vida cumplieron criterios para participar en la investigación. Cuando se les informó del estudio y se solicitó su colaboración, el $12,38 \%$ rechazó participar. De los pacientes que decidieron colaborar, entre la primera y segunda sesión el 12,38 \% de los pacientes empeoraron clínicamente, el 2,86 \% fallecie- ron y el $0,95 \%$ fueron trasladados a otro centro, por lo que finalmente la muestra está constituida por 75 pacientes, obteniéndose un índice de participación del 71,43\%.

\section{Variables e instrumentos}

Se elaboró un cuaderno de recogida de datos específico para el presente estudio, y para la valoración del malestar emocional y las estrategias de afrontamiento se utilizaron los siguientes cuestionarios: el cuestionario de Detección de Malestar Emocional (DME) ${ }^{3}$, el Mental Adjustment to Cancer Scale (MINI-MAC) ${ }^{15,16}$ y el Brief Resilient Coping Scale $(\mathrm{BRCS})^{17,18}$, respectivamente.

La historia clínica incluye los apartados de datos sociodemográficos (edad, equipo receptor, género, estado civil, estudios y red de apoyo sociofamiliar), datos clínicos (fecha de diagnóstico y tipo, sintomatología física controlada con dos opciones de respuesta) y una pregunta abierta ("¿Qué es lo más le ayuda a afrontar esta situación?"). Las respuestas a esta última pregunta se agruparon en ocho categorías (familia y amigos, fe, factores personales, hobbies, experiencia previa, estrategias adaptativas, nada y otros).

El $\mathrm{DME}^{3}$ consta de dos partes. La primera contiene tres preguntas dirigidas al paciente, dos de ellas en formato de escala analógica visual de 0 a 10 , en las que se evalúa el estado de ánimo y la percepción de afrontamiento de la situación, y otra que registra la presencia o ausencia de preocupaciones. La segunda parte consiste en una observación y registro por parte del profesional sanitario de la presencia de signos externos de malestar emocional. El DME permite obtener una puntuación de entre 0 y 20 formada por la suma de las puntuaciones de las respuestas a las preguntas referentes a la valoración del estado de ánimo y a la de percepción de afrontamiento de la situación, clasificando el malestar en leve (0-8), moderado (9-13) o grave (14-20). Las preguntas sobre preocupaciones o signos externos de malestar emocional permiten a los profesionales sanitarios una atención más específica.

El MINI-MAC ${ }^{15,16}$ es un instrumento de 29 ítems que evalúa las respuestas cognitivas y conductuales del paciente oncológico ante la enfermedad mediante una escala Likert de 4 puntos y que diferencia cinco estilos de afrontamiento: espíritu de lucha, indefensión, preocupación ansiosa, fatalismo y evitación. La estrategia de afrontamiento con una puntuación más elevada permite conocer la estrategia predominante utilizada por cada paciente.

El BRCS ${ }^{17,18}$ es una escala breve de resiliencia que consta de cuatro ítems con cinco categorías de respuesta tipo Likert. La puntuación total fluctúa entre 4 y 20 . Según los autores, una puntuación total igual o inferior a 13 indicaría baja resiliencia, mientras que puntuaciones iguales o superiores a 17 serían tributarias de alta resiliencia.

\section{Procedimiento}

Los pacientes derivados para atención psicológica, que cumplían los criterios de inclusión para formar parte del estudio, eran informados por la psicóloga del equipo correspondiente de la realización de esta investigación, así como 
de sus características y objetivos, y, por último, se preguntaba al paciente si deseaba participar. Esto se realizaba entre la primera y segunda sesión de apoyo psicológico. Si el paciente aceptaba colaborar, firmaba el consentimiento informado. A continuación, en primer lugar se recogían los datos sociodemográficos y clínicos, y posteriormente se administraban los cuestionarios: DME, MNI-MAC y BRCS, en el mismo orden. Por último, se realizaba la pregunta abierta: "¿Qué es lo que más le ayuda a afrontar esta situación?”, planteándose de la siguiente forma: "Y teniendo en cuenta todas las respuestas que nos ha proporcionado en los cuestionarios, ¿qué es lo que más le ayuda a afrontar la situación de enfermedad que está viviendo actualmente?". La entrevista tenía una duración aproximada de 30 minutos.

El estudio fue aprobado por el Comité de Ética de Investigación Científica de la Unió Catalana d'Hospitals.

\section{Análisis estadístico}

El análisis estadístico se realizó mediante el IBM SPSS Statistics versión 19. Para el análisis descriptivo de las variables cuantitativas se calculó la media, la desviación típica (SD) y el rango (puntuaciones mínimas y máximas). Se calcularon las frecuencias para describir las variables categóricas. Se realizaron pruebas de Chi cuadrado para relacionar variables categóricas y la prueba exacta de Fischer cuando no se cumplían las condiciones de aplicación. Para estudiar la relación entre variables cuantitativas se aplicó la T de Student de comparación de medias, y cuando las variables no cumplían los supuestos de normalidad se utilizó la prueba no paramétrica U de Mann-Whitney. El nivel de significación empleado en el estudio fue de $\square=0,05$.

\section{Resultados}

La muestra está constituida por 75 pacientes que presentan una edad media de 71,25 años (42-94 años) y una SD de 12,5. Más de la mitad de los pacientes son mujeres $(58,7 \%)$. Prácticamente la mitad de los enfermos están casados $(42,7 \%)$ y el $85,3 \%$ consideran que tienen apoyo sociofamiliar. El 30,7 \% tienen estudios primarios y el $28 \%$ estudios medios y superiores. Prácticamente la totalidad de la muestra son pacientes oncológicos $(86,7 \%)$ y consideran que la sintomatología física está controlada (76\%). El 53,3\% de los pacientes fueron atendidos en el domicilio y el $46,7 \%$ en un centro hospitalario (Tabla I).

El 73,3\% de los pacientes en situación de final de vida presentaban malestar emocional, siendo grave en el 29,3\% de los casos. En la Tabla II se muestra la puntuación media del DME por equipo, pacientes atendidos en el domicilio versus enfermos atendidos en un centro hospitalario. Aunque los pacientes ingresados muestran una puntuación más baja en malestar emocional comparado con los pacientes atendidos en domicilio, estas diferencias no son estadísticamente significativas.

Respecto a las preocupaciones, el 81,3\% de los pacientes expresaron tener preocupaciones. Casi la mitad, un 47,5\%, muestran preocupaciones familiares, y un 24,6 \% preocupaciones somáticas relacionadas con la enfermedad. En la Tabla III se muestran las distribuciones de las preocupacio-
Tabla I. Variables sociodemográficas y clínicas de la muestra $(n=75)$

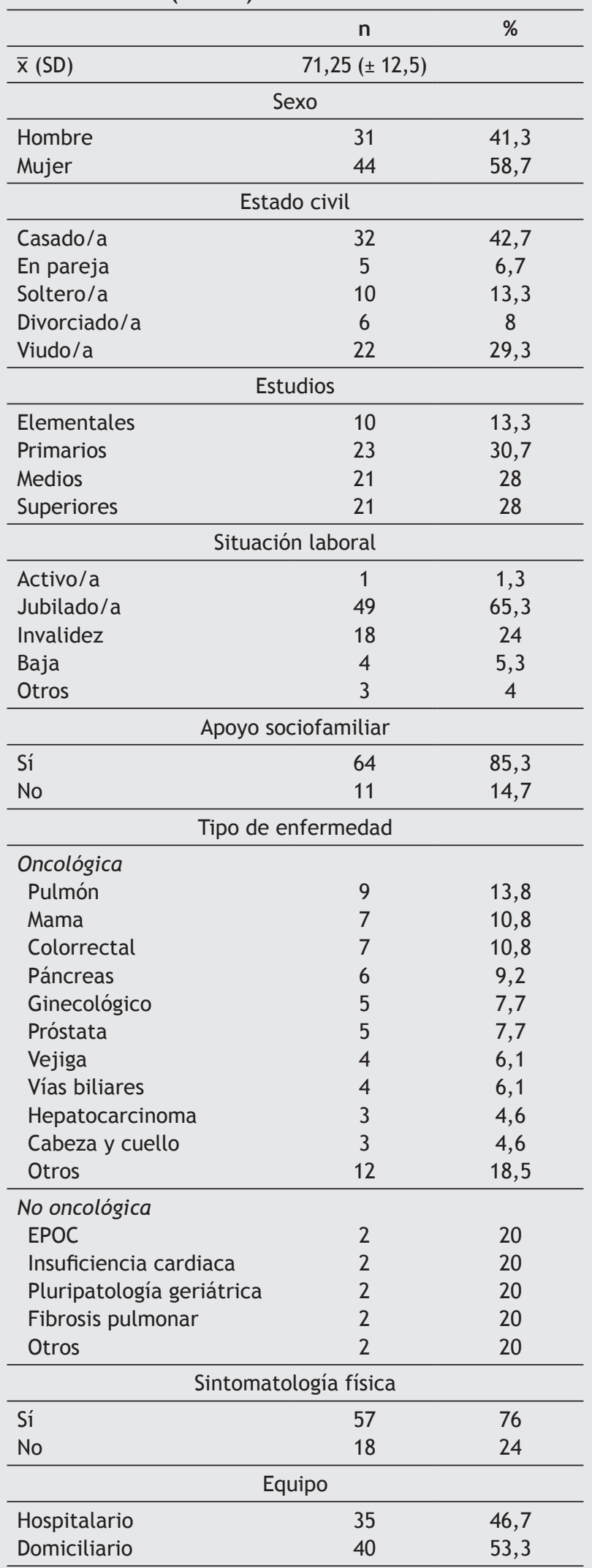

EPOC: enfermedad pulmonar obstructiva crónica. SD: desviación típica. 
Tabla II. Malestar emocional de los pacientes hospitalarios $(n=35)$ versus pacientes domiciliarios $(n=40)$

\begin{tabular}{lccc}
\hline & $\begin{array}{c}\text { Domicilio } \\
(\mathrm{n}=40) \\
\overline{\mathrm{x}}(\mathrm{SD})\end{array}$ & $\begin{array}{c}\text { Centro } \\
\text { hospitalario } \\
(\mathrm{n}=35)\end{array}$ & $p$ \\
& $\begin{array}{c}\overline{\mathrm{x}}(\mathrm{SD}) \\
\text { Rango }\end{array}$ & \\
\hline $\begin{array}{l}\text { Rango } \\
\text { emocional } \\
\text { (DME) }\end{array}$ & $\begin{array}{c}\text { 11,48 }( \pm 3,84) \\
0-20\end{array}$ & $\begin{array}{c}9,63( \pm 5,11) \\
0-20\end{array}$ & $0,149^{*}$ \\
\hline
\end{tabular}

* U de Mann-Whitney.

DME: cuestionario de Detección de Malestar Emocional.

Tabla III. Preocupaciones de los pacientes hospitalarios $(n=26)$ versus pacientes domiciliarios $(n=35)$

\begin{tabular}{lccc}
\hline Preocupaciones & $\begin{array}{c}\text { Domicilio } \\
(\mathrm{n}=40)\end{array}$ & $\begin{array}{c}\text { Centro } \\
\text { hospitalario } \\
(\mathbf{n}=35)\end{array}$ & $p$ \\
\hline Familiares & $40 \%$ & $57,7 \%$ & $0,171^{*}$ \\
\hline Somáticas & $22,9 \%$ & $26,9 \%$ & $0,715^{*}$ \\
\hline Emocionales & $8,6 \%$ & $3,8 \%$ & $0,629^{* *}$ \\
\hline Espirituales & $11,4 \%$ & - & $0,129^{* *}$ \\
\hline Otras & $17,1 \%$ & $11,5 \%$ & $0,720^{* *}$ \\
\hline
\end{tabular}

* Chi cuadrado. ${ }^{* *}$ Prueba exacta de Fisher.

nes por equipo, sin encontrarse diferencias estadísticamente significativas entre ambos grupos.

Las estrategias de afrontamiento más utilizadas por los pacientes en la fase de final de vida fueron el espíritu de lucha $(45,9 \%)$, la resiliencia (44\%) y la preocupación ansiosa (28\%). Cuando se compararon ambos grupos, los pacientes atendidos en el domicilio mostraron puntuaciones más elevadas y estadísticamente significativas en las estrategias de afrontamiento de preocupación ansiosa y desesperanza que los pacientes atendidos en un centro hospitalario (Tabla IV).

Ante la pregunta "¿Qué es lo que más le ayuda a afrontar esta situación?", prácticamente la mitad de los pacientes consideraron que el entorno familiar y social (45,3\%), seguido de las variables personales (20\%) como el carácter, las ganas de luchar, etc.

Por último, se establecieron correlaciones entre el malestar emocional y las estrategias de afrontamiento. Los resultados mostraron correlaciones positivas moderadas significativas entre el malestar emocional y las estrategias de afrontamiento desadaptativas (preocupación ansiosa $[r=0,684 ; p=0,000]$ y desesperanza $[r=0,542 ; p=0,000])$, y correlaciones negativas moderadas y moderada-baja estadísticamente significativas entre el malestar emocional y las estrategias de afrontamiento adaptativas (espíritu de lucha $[r=-0,287 ; p=0,013]$, fatalismo $[r=-0,350 ; p=0,002]$ y resiliencia $[r=-0,594 ; p=0,000])$.
Tabla IV. Estrategias de afrontamiento de los pacientes hospitalarios $(n=35)$ versus pacientes domiciliarios $(n=40)$

\begin{tabular}{|c|c|c|c|}
\hline $\begin{array}{l}\text { Estrategias de } \\
\text { afrontamiento }\end{array}$ & $\begin{array}{c}\text { Domicilio } \\
(n=40) \\
\bar{x}(S D) \\
\text { Rango }\end{array}$ & $\begin{array}{c}\text { Centro } \\
\text { hospitalario } \\
(\mathrm{n}=35) \\
\overline{\mathrm{X}}(\mathrm{SD}) \\
\text { Rango }\end{array}$ & $P$ \\
\hline $\begin{array}{l}\text { Espíritu de } \\
\text { lucha }\end{array}$ & $\begin{array}{c}9,4( \pm 4,15) \\
4-23\end{array}$ & $\begin{array}{c}10( \pm 4,35) \\
4-20\end{array}$ & $0,520^{* *}$ \\
\hline Desesperanza & $\begin{array}{c}16,92( \pm 5,32) \\
4-29\end{array}$ & $\begin{array}{c}13,41( \pm 4,94) \\
5-26\end{array}$ & $0,005^{*}$ \\
\hline $\begin{array}{l}\text { Preocupación } \\
\text { ansiosa }\end{array}$ & $\begin{array}{c}20,7( \pm 5,67) \\
10-30\end{array}$ & $\begin{array}{c}17,3( \pm 5,87) \\
8-29 \\
\end{array}$ & 0,016 * \\
\hline $\begin{array}{l}\text { Evitación } \\
\text { cognitiva }\end{array}$ & $\begin{array}{c}9,62( \pm 2,9) \\
4-16\end{array}$ & $\begin{array}{c}8,97( \pm 3,46) \\
4-21 \\
\end{array}$ & $0,218^{* *}$ \\
\hline Fatalismo & $\begin{array}{c}12,45( \pm 2,8) \\
6-17\end{array}$ & $\begin{array}{c}13,11( \pm 4,33) \\
5-20\end{array}$ & $0,441^{*}$ \\
\hline Resiliencia & $\begin{array}{c}12,6( \pm 3,56) \\
6-20\end{array}$ & $\begin{array}{c}13,03( \pm 4,45) \\
4-20\end{array}$ & $0,646^{*}$ \\
\hline
\end{tabular}

\section{Discusión}

La mayoría de los pacientes paliativos presentan malestar emocional y preocupaciones en torno a esta situación; a pesar de ello, la mayor parte de ellos utilizan estrategias de afrontamiento adaptativas como son el espíritu de lucha y la resiliencia.

El afrontamiento del final de vida genera malestar emocional, independientemente de donde han sido atendidos los pacientes, como muestra nuestro estudio y en la línea de otros trabajos realizados anteriormente ${ }^{19-21}$. Y, al mismo tiempo, los pacientes que presentan malestar emocional tienen más preocupaciones ${ }^{3,22}$.

Según el estudio de Tang y cols. ${ }^{23}$, la prevalencia de síntomas depresivos graves aumentaba a medida que se acercaba la muerte, y la probabilidad era más elevada entre aquellos pacientes con más malestar físico y dependencia funcional, los cuales recibían más apoyo de tipo práctico y expresaban más sentimientos de ser una carga, y más baja entre aquellos enfermos que manifestaban niveles más elevados de apoyo afectivo.

En otros estudios ${ }^{20,21}$, en los cuales se valoró el malestar emocional y la calidad de vida de los pacientes atendidos en el domicilio, el $25 \%$ de los pacientes mostraron niveles de ansiedad clínicamente relevantes y el $50 \%$ presentaron depresión clínica, encontrándose que esta sintomatología ansioso-depresiva disminuía la calidad de vida. Otros factores relevantes que influían en el grado de malestar fueron la carga económica y la falta de apoyo social ${ }^{24,25}$.

En un estudio reciente de Nipp y cols. ${ }^{26}$, realizado con 1.152 pacientes terminales ingresados, los autores encontraron que el $29,8 \%$ y el $28 \%$ de los pacientes presentaban síntomas ansiosos y depresivos, respectivamente, lo que estaba asociado con un mayor número de días de ingreso hospitalario y la presencia de síntomas físicos. 
Respecto a las preocupaciones de nuestros pacientes, los resultados también van en la línea de trabajos realizados en nuestro entorno. Rastero y cols. ${ }^{27}$ hallaron que el $87,76 \%$ de los pacientes en la fase final de vida expresaban preocupaciones, siendo en el $34,7 \%$ de los casos familiares y en el 28,6 \% somáticas, encontrándose una relación significativa entre la presencia de preocupaciones y la ansiedad.

Generalmente, las preocupaciones de los pacientes estaban relacionadas con la familia, concretamente con la carga que suponía la enfermedad para los diferentes miembros familiares, las preocupaciones respecto al futuro de sus hijos y/o nietos, despedirse de ellos, etc.; por otra parte, en nuestro estudio, esta variable es lo que más les ayudó a afrontar la situación de enfermedad. De hecho, el 85,3\% de los pacientes consideraban que disponían de este apoyo. En una revisión realizada por McPherson y cols. ${ }^{28}$ la percepción de ser una carga se presentaba como un problema significativo para el 19-65\% de los enfermos terminales. En nuestros pacientes podría ser posible que esta preocupación fuera lo que les proporcionaba fuerzas para luchar. Por lo tanto, el entorno familiar desempeña un papel importante en la mejora de la calidad de vida y adaptación del paciente al final de la vida.

Los pacientes del presente trabajo mostraron estrategias de afrontamiento adaptativas. A pesar de que son enfermos derivados por la detección de malestar emocional, sorprende la cantidad de personas que utilizaron estrategias adaptativas. Las variables personales fueron el segundo principal factor que ayudó a nuestros pacientes a afrontar la situación, lo cual refleja la presencia de un locus de control interno entre los enfermos atendidos que podría explicar los resultados obtenidos.

Los pacientes atendidos en el domicilio presentan puntuaciones significativamente más elevadas en las estrategias de preocupación ansiosa y desesperanza respecto a los pacientes ingresados en un centro hospitalario. Ello podría ser debido a la percepción de los pacientes de que la atención recibida en el domicilio no es la misma que podrían recibir en un hospital, donde los síntomas pueden ser más controlados y de forma más continua, generando la atención hospitalaria una percepción de mayor control de su enfermedad y proporcionando un sentimiento de mayor seguridad.

Las personas que utilizaron estrategias de afrontamiento adaptativas, es decir, espíritu de lucha y fatalismo, presentaban menos malestar emocional. La revisión de la bibliografía apoya estos resultados: aquellos pacientes que utilizaron estrategias de afrontamiento adaptativas ${ }^{29-33}$ presentaban menos malestar emocional que aquellos que tenían un estilo de afrontamiento desadaptativo ${ }^{34-36}$. Por ello podemos concluir que en este estudio la presencia de malestar emocional modula el uso de determinadas estrategias de afrontamiento, es decir, no tener malestar emocional está relacionado con estrategias de afrontamiento adaptativas.

En un estudio de Thompson y cols. ${ }^{37}$, realizado con 381 pacientes oncológicos paliativos, los autores encontraron que el $74 \%$ manifestaban aceptar la situación de final de vida. En comparación con aquellos enfermos que no aceptaban morir, una menor proporción de ellos presentaban sufrimiento y desesperanza y no cumplían con un diagnóstico de ansiedad y/o depresión. Por tanto, concluyen que la no aceptación de esta fase de la vida está asociada a sentimientos de desesperanza, sufrimiento, depresión y ansiedad.
Una posible línea de investigación futura podría ser explorar qué tipo de intervención psicológica específica podría ser más eficaz para desarrollar un estilo de afrontamiento más adaptativo, de forma que disminuyera el malestar emocional y mejorase la calidad de vida de los pacientes en los últimos días de vida. Teniendo en cuenta que la familia es la principal preocupación de los pacientes en esta fase de la enfermedad, otra línea de investigación podría ser conocer el impacto emocional que tiene esta situación en la familia, sus estrategias de afrontamiento y necesidades.

Las principales limitaciones de nuestro estudio fueron, por una parte, la dificultad en el reclutamiento de pacientes debido a que muchos de ellos no tenían información sobre su pronóstico y, por otra, se visitaron únicamente aquellos pacientes que el equipo receptor derivaba por detección de malestar emocional, o que se podían beneficiar de una intervención psicológica.

\section{Conclusiones}

Conocer el estado emocional de los pacientes y las estrategias de afrontamiento que utilizan ante la situación de final de vida permitirá desarrollar una atención psicológica preventiva con el objetivo de facilitar la adaptación a esta fase de la enfermedad. Asimismo, las preocupaciones de los pacientes están relacionadas principalmente con la familia, y al mismo tiempo es lo que más les ayuda a afrontar la enfermedad. Por lo tanto, el entorno familiar desempeña un papel muy importante en la mejora de la calidad de vida y adaptación al final de la vida. Facilitar apoyo a las familias y proporcionarles recursos, estrategias y formación en los cuidados al final de la vida podría mejorar el acompañamiento del paciente, disminuyendo la percepción de sobrecarga de este.

\section{Conflicto de intereses}

Los autores declaran no tener ningún conflicto de intereses.

\section{Financiación}

Estudio financiado por Fundació Mutuam Conviure. El trabajo recibió el Premio Fundació Conviure en el año 2015.

\section{Agradecimientos}

A todos los pacientes que han participado y aquellos que no pudieron llegar a participar en el estudio. Gracias por darnos una lección de vida en sus últimos días.

\section{Bibliografía}

1. Tang ST, Chen JS, Chou WC, Chang WC, Wu CE, Hsieh CH, et al. Longitudinal analysis of severe anxiety symptoms in the last year of life among patients with advanced cancer: relationship with proximity to death, burden, and social support. J Natl Compr Canc Netw. 2016;14:727-34. 
2. Vehling S, Malfitano C, Shnall J, Watt S, Panday T, Chiu A, et al. A concept map of death-related anxieties in patients with advanced cancer. BMJ Support Palliat Care. 2017;7:427-34.

3. Limonero JT, Mateo D, Maté J, González J, Bayés R, Bernaus M, et al. Evaluación de las propiedades psicométricas del cuestionario de Detección de Malestar Emocional (DME) en pacientes oncológicos. Gac Sanit. 2012;26:145-52.

4. Meggiolaro E, Berardi MA, Andritsch E, Nanni MG, Sirgo A, Samori E, et al. Cancer patients' emotional distress, coping styles and perception of doctor-patient interaction in European cancer settings. Palliat Support Care. 2016;14:204-11.

5. Rattner M, Berzoff J. Rethinking suffering: allowing for suffering that is intrinsic at end of life. J Soc Work End Life Palliat Care. 2016;12:240-58.

6. Soto-Rubio A, Pérez-Marín M, Barreto P. Frail elderly with and without cognitive impairment at the end of life: Their emotional state and the wellbeing of their family caregivers. Arch Gerontol Geriatr. 2017;73:113-9.

7. Washington KT, Wilkes CM, Rakes CR, Otten SJ, Parker Oliver D, Demiris $\mathrm{G}$. Relationships among symptom management burden, coping responses, and caregiver psychological distress at end of life. J Palliat Med. 2018;21:1234-41.

8. Sumpio C, Jeon S, Northouse LL, Knobf MT. Optimism, symptom distress, illness appraisal, and coping with advanced-stage cancer diagnoses undergoing chemotherapy treatment. Oncol Nurs Forum. 2017;44:384-92.

9. Shinn EH, Taylor CL, Kilgore K, Valentine A, Bodurka DC, Kavanagh J, et al. Associations with worry about dying and hopelessness in ambulatory ovarian cancer patients. Palliat Support Care. 2009;7:299-306.

10. Brown AJ, Thaker PH, Sun CC, Urbauer DL, Bruera E, Bodurka DC, et al. Nothing left to chance? The impact of locus of control on physical and mental quality of life in terminal cancer patients. Support Care Cancer. 2017;25:1985-91.

11. Lazarus RS, Folkman S. Stress, appraisal and coping. $1 .^{a}$ ed. New York: Springer Publishing Company; 1984.

12. Hopp, FP, Thornton N, Martin L. The lived experience of heart failure at the end of life: a systematic literature review. Health Soc Work. 2010;35:109-17.

13. López-Navas A, Ríos A, Martínez-Alarcón L, Febrero B, Pons JA, Miras $M$, et al. Patients with terminal chronic liver pathology faced with this disease. Transplant Proc. 2013;45:3630-2.

14. Wise M, Marchand L. Living fully in the shadow of mortal time: psychosocial assets in advanced cancer. J Palliat Care. 2013;29:76-82.

15. Román JP, Krikorian A, Palacio C. Afrontamiento del cáncer: adaptación al español y validación del Mini-MAC en población colombiana. Avances Psicol Latinoamericana. 2015;33:531-44.

16. Watson M, Law MG, dos Santos M, Greer S, Baruch J, Bliss J. The MINI-MAC. Further development of the Mental Adjustment to Cancer Scale. J Psychosoc Oncol. 1994;12:33-46.

17. Limonero JT, Tomás-Sábado J, Fernández-Castro J, GómezRomero MJ, Ardilla-Herrero A. Estrategias de afrontamiento resilientes y regulación emocional: predictores de satisfacción con la vida. Behavioral Psychology/Psicología Conductual. 2012;20:183-96.

18. Sinclair V, Wallston K. The development and psychometric evaluation of the Brief Resilient Coping Scale. Assessment. 2004;11:94-101.

19. Delgado-Guay M, Parsons HA, Li Z, Palmer JL, Bruera E. Symptom distress in advanced cancer patients with anxiety and depression in the palliative care setting. Support Care Cancer. 2009;17:573-9.

20. Krikorian A, Limonero JT. Factores asociados a la experiencia de sufrimiento en pacientes con cáncer avanzado. Avances Psicología Latinoamericana. 2015;33:423-38.
21. Rhondali W, Yennurajaligam S, Ferrer J, Chisholm G, Filbet M, Bruera E. Association between supportive care interventions and patient self-reported depression among advanced cancer outpatients. Support Care Cancer. 2014;22:871-9.

22. Enero, C, Requena M, Casas C, Gutiérrez F, Torres F, Aguirre L, et al. Relación entre preocupaciones y variables asociadas al riesgo de distress en pacientes con enfermedad oncológica avanzada. XI Congreso Nacional de Cuidados Paliativos, Sevilla 112, 13 y 14 de mayo de 2016.

23. Tang ST, Chen JS, Chou WC, Lin KC, Chang WC, Hsieh CH, et al. Prevalence of severe depressive symptoms increases as death approaches and is associated with disease burden, tangible social support, and high self-perceived burden to others. Support Care Cancer. 2016;24:83-91.

24. Götze H, Brähler E, Gansera L, Polze N, Köhler N. Psychological distress and quality of life of palliative cancer patients and their caring relatives during homecare. Support Care Cancer. 2014;22:2775-82.

25. Wang Y, Shen J, Xu Y. Symptoms and quality of life of advanced cancer patients at home: a cross-sectional study in Shanghai, China. Support Care Cancer. 2011;19:789-97.

26. Nipp RD, El-Jawahri A, Moran SM, D’Arpino SM, Johnson PC, Lage $\mathrm{DE}$, et al. The relationship between physical and psychological symptoms and health care utilization in hospitalized patients with advanced cancer. Cancer. 2017;123:4720-7.

27. Rastero S, Enero C, Rodríguez M, Jiménez MT, Ferrari L, Giner C, et al. Características de la preocupación en pacientes con enfermedad oncológica avanzada. XI Congreso Nacional de Cuidados Paliativos, Sevilla 12, 13 y 14 de mayo de 2016.

28. McPherson CJ, Wilson KG, Murray MA. Feeling like a burden to others: a systematic review focusing on the end of life. Palliat Med. 2007;21:115-28.

29. Kutner JS, Nowels DE, Kassner CT, Houser J, Bryant LL, Main DS. Confirmation of the "disability paradox" among hospice patients: preservation of quality of life despite physical ailments and psychosocial concerns. Palliat Support Care. 2003;1:231-7.

30. Sorato DB, Osório FL. Coping, psychopathology, and quality of life in cancer patients under palliative care. Palliat Support Care. 2015;13:517-25.

31. Van Laarhoven HW, Schilderman J, Bleijenberg G, Donders R, Vissers KC, Verhagen CA, et al. Coping, quality of life, depression, and hopelessness in cancer patients in a curative and palliative, end-of-life care setting. Cancer Nurs. 2011;34:302-14.

32. Bradley SE, Frizelle D, Johnson M. Coping with terminal illness: the experience of attending Specialist Palliative Day Care. J Palliat Med. 2010;13:1211-8.

33. Montoya-Juárez R, García-Caro MP, Campos-Calderón C, Schmidt-Rio Valle J, Gómez-Chica A, Martí-García C, et al. Psychological responses of terminally ill patients who are experiencing suffering: a qualitative study. Int J Nurs Stud. 2013;50:53-62.

34. Couper JW, Love AW, Duchesne GM, Bloch S, Macvean M, Dunai $\mathrm{JV}$, et al. Predictors of psychosocial distress 12 months after diagnosis with early and advanced prostate cancer. Med J Aust. 2010;193:S58-61.

35. Gilbar O, Or-Han K, Plivazky N. Mental adjustment, coping strategies, and psychological distress among end-stage renal disease patients. J Psychosom Res. 2005;58:471-6.

36. Modlinska A, Kowalik B, Buss T, Janiszewska J, LichodziejewskaNiemierko M. Strategy of coping with end-stage disease and cancer-related fatigue in terminally ill patients. Am J Hosp Palliat Care. 2014;31:771-6.

37. Thompson GN, Chochinov HM, Wilson KG, McPherson CJ, Chary $\mathrm{S}$, O'Shea FM, et al. Prognostic acceptance and the well-being of patients receiving palliative care for cancer. J Clin Oncol. 2009;27:5757-62. 